\title{
Was versteht man eigentlich unter sozialen Netzwerken?
}

Soziale Netzwerke sind zu unterscheiden von der gewachsenen Nähe familialer oder korporativer Bindung, aber auch von der funktionalen Schaltung formaler Organisation. In kritischen Lagen sozialer Rat- und Hilflosigkeit erscheinen die kleinen Kreise persönlicher Nähe heute oft als zu eng und zu schwach, um wirksam Hilfen zu bieten. Anderseits blockieren die immer abstrakter und komplexer werdenden Systemzwänge der Organisationsgesellschaft die sozialen Kräfte von gemeinschaftlicher Nähe und gesellschaftlicher Netze. Zu unterscheiden sind die "natural networks " einer scheinbar "gewachsenen « Gemeinschaftsbindung (etwa Verwandtschaft und Nachbarschaft) und die bewusst gestaltete Konstruktion »künstlicher « Netze auf der Basis gemeinsamer Interessen oder verbindender Betroffenheit. Anders als die »sekundären Systeme « professionell organisierter und offiziell installierter Fremdhilfe setzen Solidarstrategien der Netzwerkhilfe auf die verbindende Selbsterfahrung und Selbstverantwortung von Betroffenen und Beteiligten. Dazu bieten solidarische Netze nicht nur Hilfe, sondern auch Rat durch die Vernetzung von Wissen. Das bestätigen auch aktuelle Konzepte zum »sozialen Kapital « (i. S. v. Vertrauenskapital, Beziehungsreichtum, Solidaritäts-Ressourcen) durch soziale Netzwerke.

Neue Perspektiven der sozialen Netzwerkarbeit und Netzwerkförderung eröffnen sich heute über die neuen Medien elektronischer Kommunikation. In vielen, oft extremen Notlagen sozialer Hilf- und Ratlosigkeit, für die sich vor Ort keine Netze sozialer Nähe organisieren lassen, könnten die virtuellen Netzwerke Hilfen bieten, um eine Notgemeinschaft - auch über sozialräumliche Distanzen hinweg selbst zu organisieren. Sozialpolitische Programmatik würdigt die soziale Schutz-, Bewältigungs-, Entlastungs- und Unterstützungsfunktion lebensnaher Netze. In der Praxis bedeutet dies einen Perspektivwechsel Sozialer Arbeit vom individualisierenden Fallbezug zu sozial-aktivierender Feldorientierung. Als Handlungsorientierung bieten Netzwerkanalysen dem "professionellen Helfer als Netzwerker « einen Ansatz, die Stärken der alltäglichen Netzwerke und selbstorganisierten Lebensfelder anzuerkennen, zu fördern und neu zu beleben. Im Verantwortungshorizont kommunaler Sozialpolitik führt ein professionelles Verständnis von Sozialarbeit als »Netzwerk «-Arbeit aber auch zu Spannungen und Vermittlungen, Auseinandersetzungen und Verhandlungen zwischen dem politisch-administrativen System und den selbstaktiven Feldern der Selbsthilfe, Selbstorganisation und Selbststeuerung. Dann geht es nicht mehr nur um die einfache Vernetzung individueller Akteure (personale Netzwerke oder Gruppen), sondern auch um komplexere Netzwerke zwischen Gruppen, Organisationen und Assoziationen. Durch interorganisatorische Vernetzung entwickeln sich netzwerk-ökonomische Gegengewichte gegenüber globaler Marktmacht oder gegenüber politisch-administrativen Zentralismen. Während der Begriff des sozialen Netzwerks sich traditionell auf die interaktive Verknüpfung personaler Akteure bezieht, verweisen neue Konzepte des Cluster-Management auf die interorganisatorische Vernetzung korporativer Akteure (also Organisationen, Assoziationen und Institutionen wie Unternehmen, Verbände oder auch Netz- werke). Dabei wird regionale und zunehmend auch mediale Nähe zur vertrauensbildenden Basis.

Neben den sozialarbeiterischen Handlungskonzepten einer aktivierenden Vernetzung (Empowerment) interessieren Netzwerkökonomien und -strategien heute auch steuerungstheoretisch in ihrer Bedeutung für die nachhaltige Politik einer partizipativen und assoziativen Demokratie. Dies gilt auch für die Beschäftigungswirksamkeit kooperativer und alternativer Produktions-Netze. Dabei lassen sich genossenschaftliche Traditionen der sozialen Assoziation im Sinne der Verbindung von Netzwerken und Lernprozessen aktualisieren. Auch auf der Steuerungsebene von "Network-Society " eröffnen "Wissen-Netze " und darauf bauende "Policy-Netzwerke " eine strategische Offenheit für die Komplexität gesellschaftlicher Lernprozesse. Das Prinzip "Netzwerk « steht damit nicht nur für die assoziativen Netze sozialer Selbsthilfe, sondern auch für die strategischen Allianzen gesellschaftlicher Selbststeuerung.

Gerade in den Agenden einer aktiven Zivilgesellschaft erweist sich die Vernetzung gesellschaftspolitischer Akteure als wirksames Gegengewicht gegenüber staatlicher Steuerung. Zunehmend kommt es heute im Sinne von Public-Private-Partnership zu zukunftsweisenden Allianzen zwischen "politischadministrativen System " und den zivilen Netzen selbstorganisierten Engagements. Solche interorganisatorischen Verantwortungs-Netze haben sich wohlfahrtspolitisch im Zusammenspiel öffentlicher und privater Fürsorge bewährt.

Eckart Pankoke

Prof. Dr. Eckart Pankoke (1939-2007) war Soziologe und Sozialpädagoge. Bis zu seiner Emeritierung im Jahr 2004 war er Professor der Universität Duisburg-Essen.

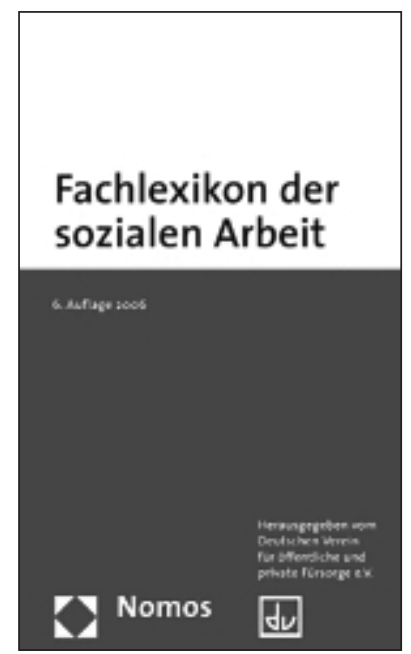

Quelle: Deutscher Verein für öfentliche und private Fürsorge e. V. (Hg.): Fachlexikon der sozialen Arbeit. 6. Auflage. Nomos Verlagsgesellschaft, Baden-Baden 2007. 1.195 Seiten. 44,- Euro. ISBN 978-38329-1825-5. Seite $857 f$. 